\title{
Seeking Darboux polynomials
}

\author{
Antoni Ferragut Armengol Gasull
}

\begin{abstract}
We introduce several techniques which allow to simplify the expression of the cofactor of Darboux polynomials of polynomial differential systems in $\mathbb{R}^{n}$. We apply these techniques to some well-known systems when $n=2,3,4$. We also propose a general method for computing Darboux polynomials in the plane. As an application we prove that a family of potential systems, that includes the van der Pol one, has no Darboux polynomials, giving in particular a new simple proof that the van der Pol limit cycle is not algebraic.
\end{abstract}

Keywords. Planar polynomial differential system, Darboux polynomial, cofactor, birational map, non-algebraic limit cycle

\section{Introduction and statement of the main results}

Consider the polynomial autonomous differential system of $\mathbb{R}^{n}$ of degree $d \in \mathbb{N}$,

$$
\dot{\mathbf{x}}=\frac{d \mathbf{x}}{d t}=P(\mathbf{x})=\left(P_{1}(\mathbf{x}), \ldots, P_{n}(\mathbf{x})\right)
$$

where $\mathbf{x}=\left(x_{1}, \ldots, x_{n}\right), P_{1}, \ldots, P_{n} \in \mathbb{R}[\mathbf{x}]$ are coprime polynomials of degree at most $d=\max \left\{\operatorname{deg} P_{1}, \ldots, \operatorname{deg} P_{n}\right\}$ and $t \in \mathbb{R}$ is the independent variable. We denote by $X(\mathbf{x})=\sum_{i=1}^{n} P_{i}(\mathbf{x}) \frac{\partial}{\partial x_{i}}$ its associated vector field.

Let $f \in \mathbb{C}[\mathbf{x}]$. We say that $f$ is a Darboux polynomial of system (1.1) if there exists a polynomial $k \in \mathbb{C}[\mathbf{x}]$ of degree at most $d-1$, called the cofactor of $f$, such that

$$
X(f)=P_{1} \frac{\partial f}{\partial x_{1}}+\cdots+P_{n} \frac{\partial f}{\partial x_{n}}=k f .
$$

A. Ferragut: Institut de Matemàtiques i Aplicacions de Castelló (IMAC) and Departament de Matemàtiques, Universitat Jaume I, Edifici TI (ESTEC), Av. de Vicent Sos Baynat, s/n, Campus del Riu Sec, 12071 Castelló de la Plana, Spain; e-mail: ferragut@uji.es

A. Gasull: Departament de Matemàtiques, Universitat Autònoma de Barcelona, Edifici C, 08193 Bellaterra, Barcelona, Catalonia-Spain; e-mail: gasull@mat.uab.cat

Mathematics Subject Classification (2010): 34C05, 34A34, 34C14 
Notice that if $f$ is a Darboux polynomial with cofactor $k$ and $m \in \mathbb{N}$ then $f^{m}$ is also a Darboux polynomial and has cofactor $m k$. It is clear that when $f$ is real the set $\left\{\mathbf{x} \in \mathbb{R}^{n}\right.$ : $f(\mathbf{x})=0\}$ is invariant by the flow of (1.1).

The existence of Darboux polynomials is a very important matter in the theory of integrability. Darboux in [9] related the number of such curves to the existence of a socalled Darboux first integral in the plane. The theorem of Darboux was extended to count also the number of exponential factors, see [8]. The result was generalized to $\mathbb{C}^{n}$, see $[20,24,38]$. In particular, if system (1.1) has at least $\left(\begin{array}{c}d+n-1 \\ n\end{array}\right)+1$ irreducible Darboux polynomials, then we may construct a Darboux or Liouville first integral. We notice that recently this lower bound has been improved in [7] for sparse differential systems. To know the Darboux polynomials it is important to reduce the dimension of the space of possible cofactors and this is precisely the first aim of this paper.

We introduce in Section 2 two techniques to simplify the general expression of the cofactors of the Darboux polynomials of system (1.1). It consists on using changes of variables given by some birational maps, the so called (quasi) monomial maps, see [3, 18, 19]. These changes, together with a suitable time parametrization, transform polynomial differential systems (resp. Darboux polynomials) into polynomial differential systems (resp. Darboux polynomials). The cofactor, which is polynomial, is to be sent to a polynomial function; this fact may fix some of the coefficients of the initial cofactor to zero.

The second technique formalizes the intuitive idea that the cofactor inherits the symmetries of the vector field. More concretely, assume that system (1.1) has a linear symmetry. This happens if there exist a certain linear map $\sigma$ and a non-zero constant $C$ such that the system is invariant by the transformation $(\mathbf{x}, t) \rightarrow(\sigma(\mathbf{x}), C t)$. This fact implies that if $f$ is a Darboux polynomial, then $f \times f \circ \sigma=0$ is another one. Then, its cofactor is the sum of the cofactor of $f$ and the cofactor of $f \circ \sigma$. The set of monomials of this new cofactor may be smaller than the set of monomials of the cofactor of $f$.

In Section 2 we also compare our approach with the one of [5], which relies on a previous work of Seidenberg [35]. In that paper several results for finding the value of the cofactor of a Darboux polynomial at some singular points are obtained. This technique will be shown to be complementary to ours.

In Section 3 we shall apply these techniques to several examples in $\mathbb{R}^{n}$, for $n=2,3,4$. In all these cases, the dimension of the space of possible cofactors decreases with these methods. Here, as an example, we state our results for the polynomial differential system in $\mathbb{R}^{3}$,

$$
\dot{x}=a_{1} x+a_{2} y, \quad \dot{y}=a_{3} x+a_{4} y+a_{5} x z, \quad \dot{z}=a_{6} x y+a_{7} z,
$$

see Example 3.5 in Section 3. It includes the Lorenz system [26], the Lü system [27] and the Chen system [6], and its Darboux integrability has been studied in [25, 23, 28], respectively. The differential system studied in [39] also belongs to this family. Combining our 
techniques we prove that for finding all its Darboux polynomials we can restrict our attention to the ones having constant cofactor. Notice that, in principle, we should consider the cofactor as an arbitrary polynomial of degree one, because the system is quadratic.

Once the set of cofactors is simplified, the next step to find all the Darboux polynomials is to handle with equation (1.2). For this problem we concentrate only in the planar case. In Section 4 we develop the method introduced in [16], that deals with the case where system (1.1) has a given analytic solution of the form $y=\alpha(x)$. More specifically, we apply to any planar polynomial system (1.1) a birational change of variables which sets the line at infinity on the horizontal axis $y=0$. Once this change is performed we are under the setting considered in [16] with $\alpha(x) \equiv 0$. In fact, it can be seen that writing (1.2) as a polynomial in $y$ and then imposing that the coefficients of this polynomial vanish, we obtain a triangular system of linear ODE in the variable $x$. The polynomial solutions of these differential equations provide the Darboux polynomials of the system.

To end the paper we apply all our techniques to study the family of potential equations $\ddot{x}=V(x, \dot{x})=a x+b \dot{x}+c x^{d-1} \dot{x}$, where $a, b, c \in \mathbb{R}$ and $d \in \mathbb{N}, d>1$. Consider its associated polynomial differential system

$$
\dot{x}=y, \quad \dot{y}=a x+b y+c x^{d-1} y .
$$

It is clear that system (1.3) for $a c=0$ is a Hamiltonian system or a linear system. The following theorem will be proved in Section 5 .

Theorem 1.1. Consider system (1.3) with ac $\neq 0$. The following statements hold.

(a) If $d>2$ then system (1.3) has no Darboux polynomials.

(b) If $d=2$ then system (1.3) has Darboux polynomials if and only if $b=0$ and they are $(a+c y)^{m}, m \in \mathbb{N}$.

(c) The system has limit cycles if and only if $d \geq 3$ is odd, $a<0$ and $b c<0$. Moreover when the limit cycle exists it is unique, hyperbolic, stable and non-algebraic.

The above result recovers some of the results of [29] about the existence of Darboux polynomials, using a different and systematic approach, and moreover characterizes completely the existence of limit cycles for system (1.3). Notice that for $d=3$, it implies that the limit cycle of the van der Pol equation $\ddot{x}+x+\varepsilon\left(x^{2}-1\right)=0$, with $\varepsilon \geq 0$, is not algebraic.

\section{Preliminary results}

We start proving a folklore lemma. 
Lemma 2.1. Consider the polynomial differential system (1.1), $\dot{\mathbf{x}}=P(\mathbf{x}), \mathbf{x} \in \mathbb{R}^{n}$ and let $f$ be a Darboux polynomial of $P$ with cofactor $k$.

(a) Let $\mathbf{x}=\phi(\mathbf{u})$ be a change of variables with inverse $\mathbf{u}=\phi^{-1}(\mathbf{x})$. Then the cofactor of the new Darboux polynomial $\tilde{f}=f \circ \phi$ for $\dot{\mathbf{u}}=Q(\mathbf{u})=D \phi^{-1}(\phi(\mathbf{u})) P(\phi(\mathbf{u}))$ is $\tilde{k}=k \circ \phi$. We denote by $Y$ the vector field associated to this new differential equation.

(b) If $g$ is a Darboux polynomial for $Y$ with cofactor $L$ and $m \in \mathbb{N}$, then $\bar{f}=g^{m} \tilde{f}$ is also a Darboux polynomial for $Y$ with cofactor $m L+\tilde{k}$.

(c) After the change of time $d t / d s=\mu(\mathbf{u})$ applied to system $\dot{\mathbf{u}}=Q(\mathbf{u})$, we obtain the new system $\mathbf{u}^{\prime}=R(\mathbf{u})=\mu(\mathbf{u}) Q(\mathbf{u})$, with associated vector field $Z$. Then $\tilde{f}$ is a Darboux polynomial for $Z$, with cofactor $\mu \tilde{k}$.

Proof. After the change of variables $\mathbf{x}=\phi(\mathbf{u})$ we obtain the new system

$$
\dot{\mathbf{u}}=Q(\mathbf{u})=D \phi^{-1}(\phi(\mathbf{u})) P(\phi(\mathbf{u})) .
$$

We show that $\tilde{f}$ is a Darboux polynomial for $Y$ with cofactor $\tilde{k}$ :

$$
\begin{aligned}
Y(\tilde{f}(\mathbf{u})) & =Y(f \circ \phi(\mathbf{u}))=\frac{d}{d t} f(\phi(\mathbf{u}))=\frac{d}{d t} f(\mathbf{x})=X(f(\mathbf{x}))=k(\mathbf{x}) f(\mathbf{x}) \\
& =k(\phi(\mathbf{u})) f(\phi(\mathbf{u}))=\tilde{k}(\mathbf{u}) \tilde{f}(\mathbf{u}) .
\end{aligned}
$$

This proves (a). To prove (b) we just need to compute $Y(\bar{f})$ :

$$
\begin{aligned}
Y(\bar{f}) & =Y\left(g^{m} \tilde{f}\right)=Y\left(g^{m}\right) \tilde{f}+g^{m} Y(\tilde{f})=m g^{m-1} Y(g) \tilde{f}+g^{m} \tilde{k} \tilde{f} \\
& =m g^{m} L \tilde{f}+g^{m} \tilde{k} \tilde{f}=(m L+\tilde{k}) g^{m} \tilde{f}=(m L+\tilde{k}) \bar{f} .
\end{aligned}
$$

To prove (c) notice that the expression of $\tilde{f}$ remains the same but the cofactor changes:

$$
Z(\tilde{f}(\mathbf{u}))=\mu(\mathbf{u}) Y(\tilde{f}(\mathbf{u}))=\mu(\mathbf{u}) \tilde{k}(\mathbf{u}) \tilde{f}(\mathbf{u})
$$

Subsection 2.1 explains how to use monomial maps to simplify the possible cofactors while Subsection 2.2 shows how to simplify them when dealing with systems exhibiting some symmetries. 


\subsection{The monomial maps}

Let $A=\left(a_{i j}\right)_{i, j=1}^{n}$ be a $n \times n$ non-singular integer matrix with $\operatorname{det} A= \pm 1$ and consider the so-called monomial map $g_{A}$ given by

$$
\mathbf{y}=\left(y_{1}, \ldots, y_{n}\right)=g_{A}(\mathbf{x})=\mathbf{x}^{A}=\left(\prod_{j} x_{j}^{a_{1 j}}, \ldots, \prod_{j} x_{j}^{a_{n j}}\right),
$$

where $\mathbf{x}=\left(x_{1}, \ldots, x_{n}\right)$. Set $A^{-1}=\left(b_{i j}\right)_{i, j=1}^{n}$. It can be seen, see for instance $[3,18,19]$, that $g_{A}$ is birational and moreover $g_{A}^{-1}=g_{A^{-1}}$, where

$$
\mathbf{x}=g_{A^{-1}}(\mathbf{y})=\mathbf{y}^{A^{-1}}=\left(\prod_{j} y_{j}^{b_{1 j}}, \ldots, \prod_{j} y_{j}^{b_{n j}}\right) .
$$

Following Lemma 2.1, we apply the change of variables (2.1) to system (1.1) to obtain, in coordinates $\mathbf{y}$,

$$
\dot{y}_{1}=\sum_{j=1}^{n} a_{1 j} \frac{y_{1} P_{j}\left(\mathbf{y}^{A^{-1}}\right)}{\prod_{k=1}^{n} y_{k}^{b_{j k}}}, \ldots, \dot{y}_{n}=\sum_{j=1}^{n} a_{n j} \frac{y_{n} P_{j}\left(\mathbf{y}^{A^{-1}}\right)}{\prod_{k=1}^{n} y_{k}^{b_{j k}}} .
$$

It may happen that we need to apply a change of time of the form

$$
\frac{d t}{d \tau}=y_{1}^{\alpha_{1}} \cdots y_{n}^{\alpha_{n}}
$$

where $\alpha_{1}, \ldots, \alpha_{n} \in \mathbb{Z}$ and $\tau$ is a new parametrization of the time, to obtain a coprime polynomial differential system:

$$
y_{1}^{\prime}=\prod_{i=1}^{n} y_{i}^{\alpha_{i}} \sum_{j=1}^{n} a_{1 j} \frac{y_{1} P_{j}\left(\mathbf{y}^{A^{-1}}\right)}{\prod_{k=1}^{n} y_{k}^{b_{j k}}}, \ldots, y_{n}^{\prime}=\prod_{i=1}^{n} y_{i}^{\alpha_{i}} \sum_{j=1}^{n} a_{n j} \frac{y_{n} P_{j}\left(\mathbf{y}^{A^{-1}}\right)}{\prod_{k=1}^{n} y_{k}^{b_{j k}}}
$$

Let $f(\mathbf{x})$ be a Darboux polynomial of system (1.1) with cofactor $k(\mathbf{x})$. Then

$$
F(\mathbf{y})=\prod_{i=1}^{n} y_{i}^{\beta_{i}} f\left(\mathbf{y}^{A^{-1}}\right)
$$

is a Darboux polynomial of system (2.4), where we choose $\beta=\left(\beta_{1}, \ldots, \beta_{n}\right) \in \mathbb{Z}^{n}$ in such that a way that $F$ is polynomial in $\mathbf{y}$ and moreover $y_{i} \nmid F$ for all $i$. After Lemma 2.1 the cofactor of $F$ writes as

$$
K(\mathbf{y})=\sum_{i=1}^{n} \beta_{i} \frac{y_{i}^{\prime}}{y_{i}}+\prod_{i=1}^{n} y_{i}^{\alpha_{i}} k\left(\mathbf{y}^{A^{-1}}\right) .
$$

We notice that in the above expression and below we write $y_{i}^{\prime}$ as a short way for denoting the polynomials appearing in the righthand sides of system (2.4). 
The denominator of $K(\mathbf{y})$ is formed only by powers of $y_{i}$. These powers of $y_{i}$ should divide $F$, as $y_{i}^{\prime}$ and $F$ are polynomials and $Y(F)=\sum_{i=1}^{n} F_{y_{i}} y_{i}^{\prime}=K F$, where we name $Y$ the vector field associated to (2.4). This is not possible according to the choice of $\beta$, therefore $K(\mathbf{y})$ must be a polynomial. Thus to simplify the general expression of the cofactor $k$, we may find monomial maps such that some monomials of corresponding $K$ are purely rational. The coefficients accompanying such monomials must be zero, and hence some annulation conditions on the coefficients of $k$ arise.

In order to find a convenient matrix $A$ we need to ensure that all the exponents of the variables in the expression of $y_{i}^{\prime}$ are non-negative. After these conditions, we may check whether some of the exponents of the variables in the expression of $K$ is negative. All this together implies that a linear collection of inequalities is to be solved, one for each coefficient of $k$. In Section 3 we successfully apply this approach to several systems, obtaining suitable matrices $A$.

While preparing this paper we were aware of the existence of [7] where the possible cofactors of sparse systems are simplified. Although the point of view of that paper is different, and does not use at all monomial maps, we suspect that both approaches can be related thought the quasi-homogeneous blow-up technique, see [2, 32]. From our point of view, our technique can be more convenient in higher dimensions: its difficulty in computation does not highly increase, while computing the Newton's polytope, which is essential in [7], may be more complicated.

\subsection{Reduction by existence of symmetries}

Next we introduce our second technique for simplifying the expression of the cofactor. It works when the vector field $X$ has some symmetry. We will assume that there exists a linear map (usually an involution) such that under the change of variables induced by this map the system is invariant after a constant reparametrization of the time. We have:

Lemma 2.2. Consider system (1.1) and let $\sigma$ be a linear map such that

$$
\sigma(P(\mathbf{x}))=C P(\sigma(\mathbf{x}))
$$

for some non-zero real constant $C$. Let $f(\mathbf{x})$ be a Darboux polynomial of system (1.1) with cofactor $k(\mathbf{x})$. Then:

(a) $f(\sigma(\mathbf{x}))$ is also a Darboux polynomial of (1.1) with cofactor $C k(\sigma(\mathbf{x}))$.

(b) $f(\mathbf{x}) f(\sigma(\mathbf{x}))$ is a Darboux polynomial of system (1.1) with cofactor $k(\mathbf{x})+C k(\sigma(\mathbf{x}))$ 
Proof. Write $\tilde{f}(\mathbf{x})=f(\sigma(\mathbf{x}))$. Since

$$
\begin{aligned}
X(\tilde{f}(\mathbf{x})) & =\nabla \tilde{f}(\mathbf{x}) P(\mathbf{x})=\nabla f(\sigma(\mathbf{x})) D \sigma(\mathbf{x}) P(\mathbf{x})=\nabla f(\sigma(\mathbf{x})) \sigma(P(\mathbf{x})) \\
& =C \nabla f(\sigma(\mathbf{x})) P(\sigma(\mathbf{x}))=C X(f(\sigma(\mathbf{x}))) \\
& =C k(\sigma(\mathbf{x})) f(\sigma(\mathbf{x}))=C k(\sigma(\mathbf{x})) \tilde{f}(\mathbf{x}),
\end{aligned}
$$

statement (a) of the lemma follows. The proof of (b) is straightforward.

Remark 2.3. Some immediate remarks follow from Lemma 2.2 .

(a) Notice that condition (2.6) implies that (1.1) is invariant by the transformation of variables and time, $(\mathbf{x}, t) \mapsto(\sigma(\mathbf{x}), C t)$.

(b) Sometimes the cofactor $k(\mathbf{x})+C k(\sigma(\mathbf{x}))$ has less unknowns than the original $k(\mathbf{x})$. Thus we can consider searching (non necessarily irreducible) Darboux polynomials with these simple cofactors. In particular, if the system has no Darboux polynomials with these particular cofactors, it has no Darboux polynomials at all.

(c) For $n=2$, if $f$ is a real Darboux polynomial,

$$
\left\{\mathbf{x} \in \mathbb{R}^{2}: f(\mathbf{x})=0\right\} \cap\left\{\mathbf{x} \in \mathbb{R}^{2}: \sigma(\mathbf{x})=\mathbf{x}\right\} \neq \emptyset,
$$

and this intersection is not exclusively formed by critical points, then by uniqueness of solutions of system (1.1) it holds that $f=0$ coincides with $\tilde{f}=0$. Therefore $k(\mathbf{x})=C k(\sigma(\mathbf{x}))$ and the cofactor $k$ of $f$ itself has already less monomials.

\subsection{On the values of the cofactor at simple singular points}

As we stated in the Introduction, in [5] several results about the value of the cofactor $k$ of a Darboux polynomial $f$ at a simple (finite or infinite) singular point $p$ of the system are given. Recall that at simple singular points the system has at least one nonzero eigenvalue. The idea of their results is the following. If $f(p) \neq 0$, then since $p$ is singular we must have $k(p)=0$; and if $f(p)=0$ then $k(p)$ is a linear combination, with non-negative integer coefficients, of the eigenvalues of the system at $p$. If this is applied to all the simple singular points, some amount of information on the cofactor is obtained.

On the one hand, the techniques for the reduction of the general expression of the cofactor explained In Sections 2.1 and 2.2 discern whether some coefficients of the cofactor are always zero or not. On the other hand, the method introduced in [5] computes the exact value of the cofactor at some points. Hence, they appear to be complementary methods, as we explain in next example.

Consider system (1.3). This system will be studied in Section 5. We shall prove with our approach that the cofactor of any Darboux polynomial depends only on $x$, see Example 3.4. That is, $k=\sum_{i=0}^{d-1} k_{i} x^{i}$. Since the system has three singular points, one of them 
being not simple, Theorems 13-15 of [5] can be applied only to the two simple singular points. They are the origin $O$ and the point $p=(1: 0: 0)$ (in projective coordinates) at infinity.

Once we set $k=k(x)$, we apply the results of [5]. At $p$ they provide $k_{d-1} \in\{0,1\}$, depending on whether the Darboux polynomial vanishes at $p$ or not. Concerning the origin, we obtain some different values for $k_{0}$, depending on the vanishing of the Darboux polynomial at $O$ and also on some conditions on the eigenvalues of the system at $O$. This last situation provides many subcases, depending on the relationship between $a$ and $b$.

Although (to be self-contained) we have not used them, it is clear that the above results on $k$, and particularly on $k_{d-1}$, might make the search of Darboux polynomials for system (1.3) easier. As we will see in the proof of Theorem 1.1, in the first step of our computations we simply obtain that $k_{d-1} \in \mathbb{N} \cup\{0\}$, see (5.4).

\section{Examples of reduction of the cofactor}

We apply the techniques in Subsections 2.1 and 2.2 to simplify the general expression of the cofactor of Darboux polynomials. We shall use the variables $(x, y)$ (resp. $(x, y, z)$ ) for systems in $\mathbb{R}^{2}$ (resp. $\mathbb{R}^{3}$ ). When applying changes of variables given by monomial maps, we shall use the new variables $(u, v)$ (resp. $(u, v, w)$ ) for systems in $\mathbb{R}^{2}$ (resp. $\mathbb{R}^{3}$ ).

We start with some examples of well-known polynomial differential systems in $\mathbb{R}^{2}$.

Example 3.1. We consider the Bogdanov-Takens system,

$$
\dot{x}=y, \quad \dot{y}=a_{1}+a_{2} y+x^{2}+x y,
$$

with $a_{1}, a_{2} \in \mathbb{R}$, see $[4,36]$. Suppose that it has a Darboux polynomial $f(x, y)$ with cofactor $k(x, y)=k_{0}+k_{1} x+k_{2} y$. We want in this first example to show how we can find a suitable matrix $A$ that simplifies as much as possible with our technique the expression of the cofactor. Consider the $2 \times 2$ integer matrix

$$
A=\left(\begin{array}{ll}
a_{11} & a_{12} \\
a_{21} & a_{22}
\end{array}\right) .
$$

We assume that $\operatorname{det} A=1$. Let the inverse matrix of $A$ be

$$
A^{-1}=\left(\begin{array}{ll}
b_{11} & b_{12} \\
b_{21} & b_{22}
\end{array}\right)=\left(\begin{array}{cc}
a_{22} & -a_{12} \\
-a_{21} & a_{11}
\end{array}\right) .
$$

The matrix $A$ provides the change of variables given by

$$
x_{1}=y_{1}^{b_{11}} y_{2}^{b_{12}}, \quad x_{2}=y_{1}^{b_{21}} y_{2}^{b_{22}}
$$


with inverse change

$$
y_{1}=x_{1}^{a_{11}} x_{2}^{a_{12}}, \quad y_{2}=x_{1}^{a_{21}} x_{2}^{a_{22}} .
$$

Applying to system (3.1) this change of variables and moreover a change of time of the form $d t / d \tau=y_{1}^{\alpha_{1}} y_{2}^{\alpha_{2}}$, we get

$$
\begin{aligned}
\dot{y}_{1}= & y_{1}^{\alpha_{1}} y_{2}^{\alpha_{2}}\left(a_{12} \alpha_{2} y_{1}+\alpha_{1} a_{12} y_{1}^{a_{21}+1} y_{2}^{-a_{11}}+a_{12} y_{1}^{a_{21}+2 a_{22}+1} y_{2}^{-a_{11}-2 a_{12}}\right. \\
& \left.+a_{12} y_{1}^{a_{22}+1} y_{2}^{-a_{12}}+a_{11} y_{1}^{1-a_{21}-a_{22}} y_{2}^{a_{11}+a_{12}}\right), \\
\dot{y}_{2}= & y_{1}^{\alpha_{1}} y_{2}^{\alpha_{2}}\left(\alpha_{2} a_{22} y_{2}+\alpha_{1} a_{22} y_{1}^{a_{21}} y_{2}^{1-a_{11}}+a_{22} y_{1}^{a_{21}+2 a_{22}} y_{2}^{1-a_{11}-2 a_{12}}\right. \\
& \left.+a_{22} y_{1}^{a_{22}} y_{2}^{1-a_{12}}+a_{21} y_{1}^{-a_{21}-a_{22}} y_{2}^{1+a_{11}+a_{12}}\right) .
\end{aligned}
$$

The monomials of the cofactor after the changes of variables and time that may be rational are

$$
k_{0} y_{1}^{\alpha_{1}} y_{2}^{\alpha_{2}}, \quad k_{1} y_{1}^{\alpha_{1}+a_{22}} y_{2}^{\alpha_{2}-a_{12}}, \quad k_{2} y_{1}^{\alpha_{1}-a_{21}} y_{2}^{\alpha_{2}+a_{11}}
$$

We want all the monomials in (3.3) to be polynomial and we want to check whether there exist values of $a_{i j}$ and $\alpha_{i}$ such that some monomials in (3.4) are rational and nonpolynomial. That is, we want

$\alpha_{1} \geq 0, \quad \alpha_{1}+a_{21} \geq 0, \quad \alpha_{1}-a_{21}-a_{22} \geq 0, \quad \alpha_{1}+a_{22} \geq 0, \quad \alpha_{1}+a_{21}+2 a_{22} \geq 0$, $\alpha_{2} \geq 0, \quad \alpha_{2}-a_{11} \geq 0, \quad \alpha_{2}-a_{11}-2 a_{12} \geq 0, \quad \alpha_{2}-a_{12} \geq 0, \quad \alpha_{2}+a_{11}+a_{12} \geq 0$,

and that one of the following conditions hold:

(1) $\alpha_{1}<0$, or $\alpha_{2}<0$;

(2) $\alpha_{1}+a_{22}<0$, or $\alpha_{2}-a_{12}<0$;

(3) $\alpha_{1}-a_{21}<0$ or $\alpha_{2}+a_{11}<0$.

Studying the above inequalities we get that only the third case may hold. Then we have $k_{2}=0$. We note that we can also use algebraic manipulators for studying the above inequalities.

We choose a set of values that makes $k_{2}=0$ : we apply to system (3.1) the change of variables given by the monomial map associated to the matrix

$$
A=\left(\begin{array}{cc}
-2 & 1 \\
1 & -1
\end{array}\right)
$$

We also change time taking $\alpha_{1}=\alpha_{2}=1$. We get the new coprime polynomial system

$$
u^{\prime}=u\left(1-2 u+v+a_{2} u v+a_{1} u^{2} v^{3}\right), \quad v^{\prime}=v\left(-1+u-v-a_{2} u v-a_{1} u^{2} v^{3}\right) .
$$

The curve $f(x, y)=0$ is transformed into $F(u, v)=u^{\beta_{1}} v^{\beta_{2}} f\left(u^{-1} v^{-1}, u^{-1} v^{-2}\right)=0$, for convenient $\beta_{i}$. The cofactor of $F$ is

$$
K(u, v)=\beta_{1} \frac{u^{\prime}}{u}+\beta_{2} \frac{v^{\prime}}{v}+k_{0} u v+k_{1}+\frac{k_{2}}{v} .
$$


Therefore $k_{2}=0$ and hence the cofactor of $f=0$ does not depend on $y$.

Indeed for $a_{2}=-2$ we have the Darboux polynomial $a_{1}-2 y+x^{2}=0$ with cofactor -2 , and for $a_{1}=-k_{0}^{2}$ and $a_{2}=k_{0}-1$ we have the invariant straight line $k_{0}-x-y=0$ with cofactor $k_{0}+x$, so the general expression of the cofactor cannot be simplified more.

Example 3.2. Consider the cubic differential system

$$
\dot{x}=a_{1} y+a_{2} y^{3}, \quad \dot{y}=a_{3} x+a_{4} x^{2}+a_{5} y^{2}+a_{6} x y^{2},
$$

that includes the Dolov system, see [11]. Assume that it has a Darboux polynomial $f$ with cofactor $k(x, y)=\sum_{i+j=0}^{2} k_{i, j} x^{i} y^{j}$. We note that system (3.5) is invariant by the transformation $(x, y,-t) \rightarrow(x,-y,-t)$. Therefore we can apply Lemma 2.2 with $\sigma(x, y)=(x,-y)$ and $C=-1$. We can consider the Darboux polynomial $f \times f \circ \sigma$, which has cofactor $k(x, y)-k(x,-y)$; that is, renaming, $k_{0,1} y+k_{1,1} x y$. Therefore when searching for the Darboux polynomials of system (3.2) we can assume that the cofactor has the above (simpler) expression. The Dolov system has also been studied with more detail in [14].

Example 3.3. We consider the quadratic differential system

$$
\dot{x}=-y, \quad \dot{y}=-x-c y+x^{2} .
$$

It is the one associated to the celebrated Fisher-Kolmogorov reaction-diffusion partial differential equation $u_{t}=u_{x x}+u(1-u)$ for searching travelling wave solutions, see $[13,15,17,21]$.

Suppose that system (3.6) has a Darboux polynomial $f$ with cofactor $k(x, y)=k_{0}+$ $k_{1} x+k_{2} y$. We apply to system (3.6) the change of variables given by the monomial map associated to the matrix

$$
A=\left(\begin{array}{cc}
-3 & 2 \\
1 & -1
\end{array}\right)
$$

see Subsection 2.1. We also change time taking $\alpha_{1}=\alpha_{2}=1$. We obtain the new coprime polynomial differential system

$$
u^{\prime}=u\left(2+3 u-2 c u v-2 u v^{2}\right), \quad v^{\prime}=v\left(-1-u+c u v+u v^{2}\right),
$$

and $f(x, y)$ is transformed into $F(u, v)=u^{\beta_{1}} v^{\beta_{2}} f\left(u^{-1} v^{-2}, u^{-1} v^{-3}\right)$, for convenient $\beta_{i}$. The cofactor of $F$ is

$$
\beta_{1} \frac{u^{\prime}}{u}+\beta_{2} \frac{v^{\prime}}{v}+k_{0} u v+\frac{k_{1}}{v}+\frac{k_{2}}{v^{2}} .
$$

Therefore we must have $k_{1}=k_{2}=0$ and hence the cofactor of $f=0$ is constant. This result is already proved in [15] with different methods and is the starting point for studying the Darboux polynomials of (3.6). In turns out that when $c=5 / \sqrt{6}$ the system has the 
Darboux polynomial $f=y^{2}+2 \sqrt{2 / 3}(1-x) y+2 / 3 x(1-x)^{2}$ with cofactor $k=-\sqrt{6}$. In fact, for this value of $c$, the partial differential equation has the traveling wave solution given in a closed and explicit form and found by Ablowitz and Zeppetella in [1].

Example 3.4. Consider the differential system (1.3) given in the introduction. Let

$$
A=\left(\begin{array}{cc}
-d & 1 \\
d-1 & -1
\end{array}\right)
$$

and $\alpha_{1}=\alpha_{2}=d-1$. We apply to system (1.3) the change of variables and time given by the techniques in Subsection 2.1 to obtain the coprime polynomial differential system

$$
\begin{aligned}
& u^{\prime}=u\left(1-d u+b u^{d-1} v^{d-1}+a u^{2 d-3} v^{2(d-1)}\right), \\
& v^{\prime}=v\left(-1+(d-1) u-b u^{d-1} v^{d-1}-a u^{2 d-3} v^{2(d-1)}\right),
\end{aligned}
$$

see (2.4). Let $f(x, y)$ be a Darboux polynomial of system (1.3) with cofactor

$$
k(x, y)=\sum_{i+j=0}^{d-1} k_{i, j} x^{i} y^{j} .
$$

Then $F(u, v)=u^{\beta_{1}} v^{\beta_{2}} f\left(u^{-1} v^{-1}, u^{1-d} v^{-d}\right)$, for convenient $\beta_{i} \in \mathbb{Z}$, is a Darboux polynomial of the above system with cofactor

$$
K(u, v)=\beta_{1} \frac{u^{\prime}}{u}+\beta_{2} \frac{v^{\prime}}{v}+\sum_{i+j=0}^{d-1} k_{i, j} u^{(d-1)(1-j)-i} v^{(d-1)(1-j)-i-j} .
$$

If $j=0$ then the exponents of $u$ and $v$ in the sum are both equal to $d-1-i$. Since $i \leq d-1$, they are non negative. However, if $j>0$ then the exponents of $u$ and $v$ in the sum are negative. As $K(u, v)$ is to be a polynomial, this implies that $k_{i, j}=0$ for all $j>0$. Hence $k(x, y)=\sum_{i=0}^{d-1} k_{i, 0} x^{i}$; that is, $k$ depends only on $x$. This fact will be useful in the proof of Theorem 1.1 in Section 1.3, because it simplifies the system of ODE to be solved.

Next example deals with a family of quadratic differential systems in $\mathbb{R}^{3}$.

Example 3.5. Consider

$$
\dot{x}=a_{1} x+a_{2} y, \quad \dot{y}=a_{3} x+a_{4} y+a_{5} x z, \quad \dot{z}=a_{6} x y+a_{7} z .
$$

As we have already explained in the introduction, it includes the Lorenz, Lü and Chen systems. The cofactor of any of its Darboux polynomials must be a linear polynomial $k(x, y, z)=k_{0}+k_{1} x+k_{2} y+k_{3} z$. Following Subsection 2.1, let

$$
A=\left(\begin{array}{rrr}
0 & 1 & -1 \\
1 & -2 & 1 \\
-2 & 3 & -2
\end{array}\right)
$$


be an non-singular $3 \times 3$ integer matrix. After the change of variables $(u, v, w)=(x, y, z)^{A}$ and the change of time $d t / d \tau=u v w$, we obtain a new coprime polynomial differential system in $\mathbb{R}^{3}$. The cofactor of $F=u^{\beta_{1}} v^{\beta_{2}} w^{\beta_{3}} f\left((u, v, w)^{A^{-1}}\right)$, with $\beta_{i}$ as in Subsection 2.1 , is

$$
K(u, v, w)=\beta_{1} \frac{u^{\prime}}{u}+\beta_{2} \frac{v^{\prime}}{v}+\beta_{3} \frac{w^{\prime}}{w}+k_{0} u v w+k_{1} u^{2}+k_{2} \frac{u}{v}+k_{3} \frac{1}{v} .
$$

As this is to be a polynomial, we have $k_{2}=k_{3}=0$. Therefore $k(x, y)=k_{0}+k_{1} x$.

We consider now the linear homogeneous function $\sigma(x, y, z)=(-x,-y, z)$ and $C=1$ and we apply Lemma 2.2. Then the Darboux polynomial $f \times f \circ \sigma$ has cofactor (renaming) $k(x, y, z)+k(-x,-y, z)=k_{0}$. Hence, following Subsection 2.2, when searching for Darboux polynomials for system (3.7) we can assume that the cofactor is constant.

We finally provide two examples in dimension four.

Example 3.6. The extended Fisher-Kolmogorov equation $u_{t}=-\gamma u_{x x x x}+u_{x x}+u-$ $u^{3}$, with $\gamma>0$, was proposed in [10] as a higher order model equation for physical systems that are bistable. For stationary solutions this equation can be written, after some transformations, as the polynomial differential system

$$
\dot{x}_{1}=x_{2}, \quad \dot{x}_{2}=x_{3}, \quad \dot{x}_{3}=x_{4}, \quad \dot{x}_{4}=x_{1}+q x_{3}-x_{1}^{3},
$$

with $\mathbf{x}=\left(x_{1}, x_{2}, x_{3}, x_{4}\right) \in \mathbb{R}^{4}$ and $q \in \mathbb{R}$.

Let $f$ be a Darboux polynomial of system (3.8) with cofactor an arbitrary polynomial $k$ of degree 2. Notice that this cofactor has 15 monomials. Consider the linear involution $\sigma\left(x_{1}, x_{2}, x_{3}, x_{4}\right)=\left(x_{1},-x_{2}, x_{3},-x_{4}\right)$ and $C=-1$. We can apply Lemma 2.2 and work with $f \times f \circ \sigma$, with corresponding cofactor

$$
k\left(x_{1}, x_{2}, x_{3}, x_{4}\right)=k_{1} x_{2}+k_{2} x_{4}+k_{3} x_{1} x_{2}+k_{4} x_{1} x_{4}+k_{5} x_{2} x_{3}+k_{6} x_{3} x_{4} .
$$

So we reduce the expression of the cofactor from 15 monomials to 6 . We rename $f \times f \circ \sigma$ as $f$.

Next we consider, following Subsection 2.1, the non-singular integer matrix

$$
A=\left(\begin{array}{rrrr}
-1 & 0 & 0 & 0 \\
-6 & -1 & 1 & 2 \\
0 & 1 & 0 & -1 \\
-3 & -1 & 0 & 2
\end{array}\right)
$$

After the change of variables and time $\left(y_{1}, y_{2}, y_{3}, y_{4}\right)=\left(x_{1}, x_{2}, x_{3}, x_{4}\right)^{A}, d t / d \tau=y_{1}^{2} y_{4}$, we obtain a new coprime polynomial differential system in $\mathbb{R}^{4}$. The cofactor of $F=$ 
$y_{1}^{\beta_{1}} y_{2}^{\beta_{2}} y_{3}^{\beta_{3}} y_{4}^{\beta_{4}} f\left(\mathbf{y}^{A^{-1}}\right)=0$, with $\beta_{i}$ as in Subsection 2.1, is

$$
\begin{aligned}
K\left(y_{1}, y_{2}, y_{3}, y_{4}\right)= & \beta_{1} \frac{y_{1}^{\prime}}{y_{1}}+\beta_{2} \frac{y_{2}^{\prime}}{y_{2}}+\beta_{3} \frac{y_{3}^{\prime}}{y_{3}}+\beta_{4} \frac{y_{4}^{\prime}}{y_{4}} \\
& +k_{1} \frac{y_{3}^{2} y_{4}^{2}}{y_{1}}+k_{2} \frac{y_{3} y_{4}^{2}}{y_{1}}+k_{3} \frac{y_{3}^{2} y_{4}^{2}}{y_{1}^{2}}+k_{4} \frac{y_{3} y_{4}^{2}}{y_{1}^{2}}+k_{5} \frac{y_{2} y_{3}^{2} y_{4}}{y_{1}^{4}}+k_{6} \frac{y_{2} y_{3} y_{4}}{y_{1}^{4}} .
\end{aligned}
$$

Hence, $k_{j}=0, j=1,2, \ldots, 6$. This means that if the initial system has a Darboux polynomial then it has another Darboux polynomial with null cofactor. In particular it is to be a polynomial first integral. Indeed, straightforward computations show that system (3.8) has the first integral $x_{1}^{4} / 4-\left(x_{1}^{2}+q x_{2}^{2}+x_{3}^{2}\right) / 2+x_{2} x_{4}$.

Example 3.7. Consider the Raychaudhuri polynomial system in $\mathbb{R}^{4}$

$$
\begin{aligned}
& \dot{x}_{1}=-\frac{1}{2} x_{1}^{2}-\alpha x_{1}-2\left(x_{2}^{2}+x_{3}^{2}-x_{4}^{2}\right)-2 \beta, \\
& \dot{x}_{2}=-\left(\alpha+x_{1}\right) x_{2}-\gamma \\
& \dot{x}_{3}=-\left(\alpha+x_{1}\right) x_{3}-\delta \\
& \dot{x}_{4}=-\left(\alpha+x_{1}\right) x_{4}
\end{aligned}
$$

where $\alpha, \beta, \gamma, \delta \in \mathbb{R}$. The Darboux integrability of theis system was studied in [37]. In the particular case $\gamma \neq 0$ and $\delta=0$, this system can be brought, through a linear change of variables and time, to

$$
\begin{aligned}
\dot{x}_{1} & =-\frac{1}{2} x_{1}^{2}-2 \gamma_{1} x_{2}^{2}-\frac{\gamma_{1}}{2} x_{3}^{2}-2 \gamma_{2} x_{2} x_{3}+2 x_{4}^{2}-\gamma_{3}, \\
\dot{x}_{2} & =-x_{1} x_{2}-1 \\
\dot{x}_{3} & =-x_{1} x_{3} \\
\dot{x}_{4} & =-x_{1} x_{4}
\end{aligned}
$$

where $\gamma_{1} \in \mathbb{R}^{+}$and $\gamma_{2}, \gamma_{3} \in \mathbb{R}$.

It was stated in [37] that there that if this system has an irreducible Darboux polynomial of degree at least two, then its cofactor is a multiple of $x_{1}$. But straightforward computations show that we have four Darboux polynomials

$2\left(\gamma_{3}+k_{0}^{2}\right)+2 k_{0} x_{1}-2 k_{0}\left(2 \gamma_{3}+k_{0}^{2}\right) x_{2}+4 \frac{\gamma_{2}}{k_{0}} x_{3}+x_{1}^{2}-4 \gamma_{1} x_{2}^{2}-\gamma_{1} x_{3}^{2}+4 x_{4}^{2}-4 \gamma_{2} x_{2} x_{3}$

with $k_{0}$ satisfying $4 \gamma_{1}+2 \gamma_{3} k_{0}^{2}+k_{0}^{4}=0$, of degree two with cofactor $k_{0}-x_{1}$. Moreover, we note that if we name $f_{i}, i=1,2,3,4$, the four Darboux polynomials above, we can easily construct a rational first integral $\left(f_{1} f_{2}\right) /\left(f_{3} f_{4}\right)$ of the system. These facts show that some cases in [37] are missing.

Applying our first technique it is not possible in this case to reduce the expression of the cofactor of an irreducible Darboux polynomial. Concerning the second technique, 
we apply Lemma 2.2, first with $\sigma_{1}\left(x_{1}, x_{2}, x_{3}, x_{4}\right)=\left(x_{1}, x_{2}, x_{3},-x_{4}\right)$ and $C=1$, and afterwards with $\sigma_{2}\left(x_{1}, x_{2}, x_{3}, x_{4}\right)=\left(-x_{1},-x_{2},-x_{3},-x_{4}\right)$ and $C=-1$. We recall that the cofactor of any Darboux polynomial of system (3.9) has the form $k_{0}+k_{1} x_{1}+k_{2} x_{2}+$ $k_{3} x_{3}+k_{4} x_{4}$. In the first case we remove the coefficient $k_{4}$, and in the second case we remove the coefficient $k_{0}$. Hence applying both symmetries we may consider Darboux polynomials having the cofactor $k_{1} x_{1}+k_{2} x_{2}+k_{3} x_{3}$. We do not know whether there exist Darboux polynomials having cofactor with $k_{2}, k_{3} \neq 0$.

\section{Darboux polynomials in the plane}

In [16] a method to determine the existence of Darboux polynomials for planar analytic differential system having an analytic solution of the form $y=\alpha(x)$ is introduced. Here we adapt it to general polynomial systems using the line at infinity as this known solution.

\subsection{Reduction of (1.2) to a system of ODE}

Consider the planar polynomial differential system

$$
\dot{x}=\frac{d x}{d t}=P(x, y), \quad \dot{y}=\frac{d y}{d t}=Q(x, y) .
$$

We change the variables in such a way that the line at infinity becomes the horizontal axis; that is, we take $(u, v)=(x / y, 1 / y)$. After this change of variables and the change of time $d t / d \tau=v^{d-1}$, system (4.1) becomes the polynomial differential system

$$
\begin{aligned}
& \dot{u}=X(u, v)=v^{d}\left[P\left(\frac{u}{v}, \frac{1}{v}\right)-u Q\left(\frac{u}{v}, \frac{1}{v}\right)\right], \\
& \dot{v}=Y(u, v)=-v^{d+1} Q\left(\frac{u}{v}, \frac{1}{v}\right),
\end{aligned}
$$

where the dot means now derivative with respect to $\tau$. This system has degree $d+1$. The change of variables sends the straight line $y=0$ of system (4.1) to the infinity of system (4.2), and the infinity of system (4.1) to the invariant straight line $v=0$ of system (4.2).

Let $f(x, y)$ be a Darboux polynomial of degree $m \in \mathbb{N}$ of system (4.1) with cofactor $k(x, y)$. Let $F(u, v)=v^{m} f(u / v, 1 / v)$ be the corresponding Darboux polynomial of system (4.2). Its cofactor is the polynomial $K(u, v)=m Y(u, v) / v+v^{d-1} k(u / v, 1 / v)$, see Lemma 2.1. Observe that $\operatorname{deg} K \leq d$.

We note that instead of the above change of variables one can apply the change $(u, v)=(y / x, 1 / x)$ (and the same change of time), from which we obtain a new similar polynomial differential system of degree $d+1$. 
If $F(u, v)$ is a Darboux polynomial of degree $m \in \mathbb{N}$ of system (4.2) and $K(u, v)$ is its cofactor, then the following equation holds:

$$
X(u, v) F_{u}(u, v)+Y(u, v) F_{v}(u, v)=K(u, v) F(u, v) .
$$

The analytic solution $y-\alpha(x)=0$ in [16] is here $v=0$. Therefore we write $F=$ $\sum_{i=0}^{m} F_{i}(u) v^{i}$ and $K=\sum_{i=0}^{d} K_{i}(u) v^{i}$, for some convenient polynomials $F_{i}$ and $K_{i}$. We write (4.3) in powers of $v$, so that a system of ODE in the variable $u$ with unknowns the $F_{i}$ arises. Following [16] we notice that our approach applies only when $v \nmid F(u, v)$.

Next we show how to obtain these equations in terms of the initial system. If $P(x, y)=$ $\sum_{i+j=0}^{d} p_{i j} x^{i} y^{j}$ and $Q(x, y)=\sum_{i+j=0}^{d} q_{i j} x^{i} y^{j}$, then

$$
X(u, v)=\sum_{i+j=0}^{d}\left(p_{i j}-u q_{i j}\right) u^{i} v^{d-i-j} ; \quad Y(u, v)=-\sum_{i+j=0}^{d} q_{i j} u^{i} v^{d+1-i-j} .
$$

Equation (4.3) becomes

$$
\sum_{j=0}^{m+d}\left(\sum_{i=0}^{j}\left[X_{j-i}(u) F_{i}^{\prime}(u)+\left(i Y_{j-i+1}(u)-K_{j-i}(u)\right) F_{i}(u)\right]\right) v^{j}=0,
$$

where

$$
X_{j-i}(u)=\sum_{l=0}^{d}\left(p_{l, d-l-(j-i)}-u q_{l, d-l-(j-i)}\right) u^{l}, \quad Y_{j-i+1}(u)=-\sum_{l=0}^{d} q_{l, d-l-(j-i)} u^{l} .
$$

From the above relation the functions $F_{j}(u)$ can be obtained recurrently by solving for $0 \leq j \leq m$ the linear differential equations with unknowns the $F_{j}(u)$ that arise by vanishing the coefficient of $v^{j}$ in (4.5). The general expression of these equations is

$$
X_{0}(u) F_{j}^{\prime}(u)=\left(K_{0}(u)-j Y_{1}(u)\right) F_{j}(u)+R_{j}(u)
$$

where $F_{j}$ is unknown and $R_{j}(u)=\sum_{i=0}^{j-1}\left[\left(K_{j-i}(u)-i Y_{j-i+1}(u)\right) F_{i}(u)-X_{j-i}(u) F_{i}^{\prime}(u)\right]$. In particular, for $j=0,1$, we get

$$
\begin{aligned}
& X_{0}(u) F_{0}^{\prime}(u)=K_{0}(u) F_{0}(u), \\
& X_{0}(u) F_{1}^{\prime}(u)=\left(K_{0}(u)-Y_{1}(u)\right) F_{1}(u)+K_{1}(u) F_{0}(u)-X_{1}(u) F_{0}^{\prime}(u) .
\end{aligned}
$$

In the former case this means that the primitive of $K_{0}(u) / X_{0}(u)$ must be a linear combination of logarithms of polynomials, and the coefficients of these logarithms must be non-negative integers.

As for each $j$ the function $F_{j}(u)$ must be polynomial, the linear differential equations described in (4.6) may provide a collection of necessary conditions which restrict the 
coefficients of $P, Q$ and $k$ (and perhaps also the degree of $f$ as it appears in the expression of $K)$. These restrictions give us the key for searching the algebraic solutions of system (4.2), and therefore of system (4.1), or for proving that no algebraic solutions exist. Indeed for $j=0, \ldots, m$ equation (4.6) provides the expression of $F_{j}$ and perhaps some of these conditions; and for $j=m+1, \ldots, m+d$ it provides conditions on the coefficients of $X, Y$ and $K$ and on the integration constants appearing in the expression of the $F_{i}$, as all the $F_{i}$ have already been computed.

In summary, equation (4.3) is equivalent to a set of quadratic equations where the unknowns are the coefficients of $F$ and $K$ in $v$, which are functions of $u$. The equations follow a given pattern and can be solved to obtain the coefficients of $F$ and moreover some restrictions on $K$ and on the system. In general, it is hard to solve this system of equations, even by using algebraic manipulators. Next subsection is devoted to find polynomial solutions of a linear polynomial differential equation. The results will be useful to deal with equation (4.6).

\subsection{Polynomial solutions of linear ordinary differential equations}

In this subsection we provide some common knowledge results about polynomial solutions of linear polynomial differential equations.

Given a first order linear differential equation, non-necessarily polynomial,

$$
p(u) f^{\prime}(u)=q(u) f(u)+r(u),
$$

it is easy to prove that one of the following situations holds:

- It has no polynomial solutions.

- It has exactly one polynomial solution.

- All its solutions are polynomial.

As we will see, our study of system (1.3) will need the characterization of the above third possibility. We prove the following result:

Proposition 4.1. Consider equation (4.7) with $p, q, r \in \mathbb{C}[u]$ such that $\operatorname{gcd}(p, q)=$ $\operatorname{gcd}(p, r)=1$. Suppose that $p$ is monic and that $\operatorname{deg} p=N \in \mathbb{N}$. All the solutions of (4.7) are polynomial if and only if the following conditions hold:

(a) $\operatorname{deg} q<N$.

(b) $p(u)$ is square free; i.e., $p(u)=\prod_{i=1}^{N}\left(u-u_{i}\right)$, where $u_{i} \in \mathbb{C}$ and $u_{i} \neq u_{j}$ for $i \neq j$.

(c) $q\left(u_{i}\right) / p^{\prime}\left(u_{i}\right)=n_{i} \in \mathbb{N} \cup\{0\}$, for all $i \in\{1, \ldots, N\}$. 
(d) $\phi_{i}^{\left(n_{i}\right)}\left(u_{i}\right)=0$, where $\phi_{i}(u)=r(u) / \prod_{k \neq i}\left(u-u_{k}\right)^{n_{k}+1}$, for all $i \in\{1, \ldots, N\}$.

Proof. We apply the method of variation of the constants to solve equation (4.7). First we consider the linear homogeneous differential equation

$$
p(u) f^{\prime}(u)=q(u) f(u)
$$

In order to solve this equation we write it as

$$
\frac{f^{\prime}(u)}{f(u)}=\frac{q(u)}{p(u)}
$$

Integrating with respect to $u$ the left-hand side of this equation we get $\ln |f(u)|$. Let $q_{1}, q_{2} \in \mathbb{C}[x, y]$ be such that $q(u)=q_{1}^{\prime}(u) p(u)+q_{2}(u)$, with $\operatorname{deg} q_{2}<\operatorname{deg} p$. Then

$$
\int \frac{q(u)}{p(u)} d u=q_{1}(u)+\int \frac{q_{2}(u)}{p(u)} d u .
$$

We need to obtain a linear combination of logarithms from the right-hand side of (4.9). This happens if and only if $q_{1}$ is constant, which is condition (a), and $p$ is square free, which is condition (b), as we are assuming that $(p, q)=1$. Thus $q_{2}=q$ and $q_{1}$ is the integration constant. Set $p(u)=\prod_{i=1}^{N}\left(u-u_{i}\right)$, with $u_{i} \in \mathbb{C}$. Then the set of solutions of (4.9) is

$$
f(u)=C \prod_{i=1}^{N}\left(u-u_{i}\right)^{n_{i}}
$$

for some $n_{i} \in \mathbb{C}$, being $C$ an arbitrary constant. Writing $f(u)=C(u) \prod_{i=1}^{N}\left(u-u_{i}\right)^{n_{i}}$ and searching $C(u)$ we obtain that the general solution of (4.7) is

$$
f(u)=c \prod_{i=1}^{N}\left(u-u_{i}\right)^{n_{i}}+\prod_{i=1}^{N}\left(u-u_{i}\right)^{n_{i}} \int \frac{r(u)}{\prod_{i=1}^{N}\left(u-u_{i}\right)^{n_{i}+1}} d u
$$

where $c$ is an arbitrary constant. The first summand must be a polynomial. This is equivalent to say that $n_{i} \in \mathbb{N} \cup\{0\}$ for all $i$, which is condition (c). After this observation, we can write

$$
\frac{r(u)}{\prod_{i=1}^{N}\left(u-u_{i}\right)^{n_{i}+1}}=s^{\prime}(u)+\sum_{i=1}^{N} \sum_{j=1}^{n_{i}+1} \frac{B_{i, j}}{\left(u-u_{i}\right)^{j}},
$$

where $s$ is a polynomial and $B_{i, j} \in \mathbb{C}$. We note that $s(u) \not \equiv 0$ if and only if $\operatorname{deg} r \geq$ $\sum_{i=1}^{N} n_{i}+\operatorname{deg} p$. We also recall that $\left(u-u_{i}\right) \nmid r(u)$ as $\operatorname{gcd}(p, r)=1$. Integrating the sums above, for each $j>1$ we get $B_{i, j}(1-j)\left(u-u_{i}\right)^{1-j}$, and for $j=1$ we obtain $B_{i, 1} \ln \left|u-u_{i}\right|$. Thus we must impose $B_{i, 1}=0$ for all $i \in\{1, \ldots, N\}$. This is equivalent to condition $(\mathrm{d})$. 
The expression of the solutions $f(u)$ of (4.7) is, hence,

$$
f(u)=\prod_{i=1}^{N}\left(u-u_{i}\right)^{n_{i}}\left(c+s(u)-\sum_{i=1}^{N} \sum_{j=2}^{n_{i}+1} \frac{B_{i j}(j-1)}{\left(u-u_{i}\right)^{j-1}}\right) .
$$

Remark 4.2. We state the following observations for linear equations having polynomial solutions.

(a) If $p$ and $q$ have common factors, then from (4.7) these factors also divide $r$ and hence they can be simplified. Thus the condition $(p, q)=1$ is not restrictive.

(b) If $p$ has multiple factors, say $p(u)=\prod_{i=1}^{N}\left(u-u_{i}\right)^{\alpha_{i}}$, with $\alpha_{i} \in \mathbb{N}$ and $\alpha_{i}>1$ for some $i$, then $q$ should also have these factors $u-u_{i}$ in its decomposition powered to $\alpha_{i}-1$, because of (4.8); that is, $\prod_{i=1}^{N}\left(u-u_{i}\right)^{\alpha_{i}-1} \mid q(u)$. Then from (4.7) the factors $\prod_{i=1}^{N}\left(u-u_{i}\right)^{\alpha_{i}-1}$ also divide $r$ and hence they are simplified.

(c) If $p$ and $r$ have common factors, then the quotient in the left hand side of equation (4.10) is simplified. Hence the condition (d) in the proposition varies but it is still related to vanish the coefficients of $\left(u-u_{i}\right)^{-1}$ which provide logarithms after integration.

(d) In the situation of Proposition 4.1, if conditions (a) or (d) do not hold, then equation (4.7) has no polynomial solutions, as there are non-polynomial functions in the expression of $f$ that cannot be removed. If conditions (b) or (c) do not hold, then equation (4.7) has at most one polynomial solution, as the integration constant needs to be fixed to have a polynomial solution.

In equations (4.6) we have $p=X_{0}$ and $q=K_{0}-j Y_{1}$, and $\operatorname{deg} p>\operatorname{deg} q$, hence in our situation condition (a) always holds. We also notice that $X_{0}$ may have multiple factors, but they should be canceled in equation (4.7) as we explained in Remark 4.2.

In order to obtain all the polynomial solutions of (4.3) we need for each equation (4.6) with $j \in\{0, \ldots, m\}$ to have either one or infinitely many polynomial solutions. Moreover equations (4.6) for $j \in\{m+1, \ldots, m+d\}$ also need to be satisfied.

\subsection{The case $Y_{1}(u) \equiv 0$}

We consider in this subsection the particular case $Y_{1}(u) \equiv 0$. We note that this is equivalent to $\operatorname{deg} Q<\operatorname{deg} P$, because $Y_{1}(u) v=-Q_{d}(u, 1) v$, see (4.2), where $Q_{d}$ is the homogeneous polynomial of degree $d$ of $Q$. That is, $Q_{d}(x, y)=\sum_{i=0}^{d} q_{i, d-i} x^{i} y^{d-i}$ and $Y_{1}(u)=-\sum_{i=0}^{d} q_{i, d-i} u^{i}$, see (4.4). Thus, if for a polynomial differential system there is a 
linear change of variables from which we obtain a system such that $\operatorname{deg} P \neq \operatorname{deg} Q$, then $Y_{1}(u) \equiv 0$.

If $Y_{1}(u) \equiv 0$ then equation (4.6) writes

$$
X_{0} F_{j}^{\prime}=K_{0} F_{j}+R_{j}
$$

for all $j \geq 0$. In the sequel we show that the set of conditions of Proposition 4.1 for every $j \geq 0$ is reduced.

First of all we consider equation (4.12) with $j=0$. In this equation we have $R_{0}=0$, hence

$$
F_{0}(u)=\exp \left(\int \frac{K_{0}(u)}{X_{0}(u)} d u\right)=\exp \left(\sum_{i=1}^{N} \int \frac{n_{i, 0}}{u-u_{i}} d u\right)=\prod_{i=1}^{n}\left(u-u_{i}\right)^{n_{i, 0}} .
$$

Therefore we must have $K_{0}(u)=\sum_{i=1}^{N} n_{i, 0} \prod_{j \neq i}\left(u-u_{i}\right)$ and $n_{i, 0} \in \mathbb{N} \cup\{0\}$. This means of course that $\operatorname{deg} K_{0}<\operatorname{deg} X_{0}$. Then the condition (a) of the proposition holds.

If $X_{0}$ is not square free then we shall have common multiple factors in $X_{0}$ and $K_{0}$, and hence also in $R_{j}$. These extra factors can be cancelled and hence we obtain a new equation having $X_{0}$ (renaming) square free, see Remark 4.2. This is condition (b) of the proposition.

As $Y_{1}(u) \equiv 0$, the polynomials $p(u)$ and $q(u)$ in (4.7) are always the same for equation (4.12): $p(u)=X_{0}(u)$ and $q(u)=K_{0}(u)$. Hence for all $j$ the $n_{i, j}$ are the same: $n_{i, j}=$ $n_{i, 0} \in \mathbb{N} \cup\{0\}$. Thus condition (c) of the proposition must be checked only for $j=0$. We rename $n_{i, j}=n_{i}$, for all $i, j$.

The only condition that we need to check at each step is condition (d). This implies that for all $j \geq 0$ there are either zero or infinitely many polynomial solutions $F_{j}$, as the integration constant $C_{j}$ is not fixed in any case and we can apply Proposition 4.1.

After solving equation (4.12) for $j=0, \ldots, m$, we have computed the expression of $F$. But there are also $d$ equations to be satisfied, see (4.5). These equations are not differential equations, as $F_{j}(u) \equiv 0$ for $j>m$, indeed they are $R_{j}=0$. Hence all them together can be written as a linear system of equations where the integration constants $C_{j}$ of all the $F_{j}$ are unknown and each equation corresponds to the coefficients of $R_{j}$ in $u$ equaled to zero. This system may fix some of the $C_{j}$ and give some restrictions on the coefficients of the system. If there is no compatibility, then the system has no Darboux polynomial $F$. If we have compatibility we have existence of Darboux polynomials.

Section 5 deals with a differential system for which we have $Y_{1}(u) \equiv 0$. We shall apply the results of this subsection there. 


\section{A potential system with non-algebraic limit cycles}

Consider the potential differential equation $\ddot{x}=V(x, \dot{x})$, where $V \in \mathbb{R}[x, y]$ has degree $d \in \mathbb{N}, d>1$. Let

$$
\dot{x}=y, \quad \dot{y}=V(x, y)
$$

be its associated planar differential system of degree $d$. After the change of variables $(x, y)=(1, u) / v$ and the change of time $d t / d s=v^{d-1}$ we obtain the polynomial differential system

$$
\dot{u}=X(u, v)=v^{d} V\left(\frac{u}{v}, \frac{1}{v}\right)-u^{2} v^{d-1}, \quad \dot{v}=Y(u, v)=-u v^{d},
$$

which has degree $d+1$. The dot means now derivative with respect to $s$. We note that $Y_{0}=\cdots=Y_{d-1}=0$ and $Y_{d}(u)=-u$. Since $d>1$ we have in particular $Y_{1}=0$, see Subsection 4.3.

\subsection{Proof of Theorem 1.1}

Since $c \neq 0$, after a change of time we can set $c=1$. We first prove statements (a) and (b) following the techniques of previous sections.

System (5.2) writes, for $V(x, y)=a x+b y+x^{d-1} y$, as

$$
\dot{u}=X(u, v)=u+\left(a+b u-u^{2}\right) v^{d-1}, \quad \dot{v}=Y(u, v)=-u v^{d}
$$

Thus we have

$$
\begin{aligned}
X_{0} & =u ; \quad X_{1}=\cdots=X_{d-2}=0 ; \quad X_{d-1}=a+b u-u^{2} ; \quad X_{d}=X_{d+1}=0 ; \\
Y_{0} & =\cdots=Y_{d-1}=0 ; \quad Y_{d}=-u ; \quad Y_{d+1}=0 .
\end{aligned}
$$

Let $f(x, y)$ be a Darboux polynomial of degree $m \in \mathbb{N}$ of the differential system (1.3) with cofactor $k$. We know from Example 3.4 that $k=k(x)$, hence we can write $k(x)=$ $\sum_{i=0}^{d-1} k_{i} x^{i}$. Consider the corresponding Darboux polynomial $F(u, v)=v^{m} f(1 / v, u / v)=$ $\sum_{i=0}^{m} F_{i}(u) v^{i}$ of degree $m$ of system (5.3), which has the cofactor $K(u, v)=-m u v^{d-1}+$ $\sum_{i=0}^{d-1} k_{i} v^{d-1-i}$. We write $K(u, v)=\sum_{i=0}^{d} K_{i}(u) v^{i}$, with

$$
K_{i}(u)=k_{d-1-i}, \quad K_{d-1}(u)=k_{0}-m u, \quad K_{d}(u)=0,
$$

for $i=0, \ldots, d-2$. We shall follow Proposition 4.1 to solve equation (4.3) for system (5.3). Observe that conditions (a) and (b) in Proposition 4.1 are fulfilled for all $j$.

Equation (4.6) with $j=0$ writes as

$$
u F_{0}^{\prime}=k_{d-1} F_{0}
$$


From Proposition 4.1 we must take

$$
k_{d-1}=n \in \mathbb{N} \cup\{0\} .
$$

Hence $F_{0}(u)=u^{n}$ and $K_{0}(u)=n$.

We notice that condition (d) of Proposition 4.1 is equivalent to the fact that the coefficient of $u^{n}$ in $R_{j}$ vanishes, for all $j$. Equation (4.6) with $j=1$ writes as

$$
u F_{1}^{\prime}=n F_{1}+k_{d-2} u^{n} .
$$

We must take $K_{1}(u)=k_{d-2}=0$. Then we get $F_{1}(u)=C_{1} u^{n}$, where $C_{1}$ is a constant. In a similar way, applying induction arguments, from equation (4.6) with $j=2, \ldots, d-2$ we get $K_{j}(u)=k_{d-1-j}=0$ and $F_{j}(u)=C_{j} u^{n}$, where $C_{j}$ is a constant. This is because $R_{j}=k_{d-1-j} u^{n}$. We note that at this moment we have proved that $K(u, v)=K_{0}(u)+$ $K_{d-1}(u) v^{d-1}=n+\left(k_{0}-m u\right) v^{d-1}$.

Equation (4.6) with $j=d-1$ writes as $u F_{d-1}^{\prime}=n F_{d-1}+R_{d-1}$, with

$$
R_{d-1}(u)=-a n u^{n-1}+\left(k_{0}-b n\right) u^{n}-(m-n) u^{n+1} .
$$

Therefore we must take $k_{0}=b n$, and hence $K_{d-1}(u)=b n-m u$. Moreover

$$
F_{d-1}(u)=a n u^{n-1}+C_{d-1} u^{n}-(m-n) u^{n+1},
$$

where $C_{d-1}$ is a constant.

Equation (4.6) with $j=d$ writes as $u F_{d}^{\prime}=n F_{d}+R_{d}$, with

$$
R_{d}(u)=-a n C_{1} u^{n-1}-(m+1-n) C_{1} u^{n+1} .
$$

We have $F_{d}(u)=a n C_{1} u^{n-1}+C_{d} u^{n}-(m+1-n) C_{1} u^{n+1}$, where $C_{d}$ is a constant. No new conditions need to be satisfied since $R_{d}$ has not the monomial $u^{n}$.

A similar pattern occurs for equation (4.6) for $j=d+1, \ldots, 2 d-3$. We obtain no new conditions and moreover $F_{d+i}=a n C_{i+1} u^{n-1}+C_{d+i} u^{n}-(m+i-n) C_{i+1} u^{n+1}$, for $i=1, \ldots, d-3$, where $C_{d+i}$ are constants.

We next consider equation (4.6) for $j=2 d-2$, which is $u F_{2 d-2}^{\prime}=n F_{2 d-2}+R_{2 d-2}$, with $R_{2 d-2}(u)=\cdots+(a(m+(d-3) n)) u^{n}+\cdots$. Since $a \neq 0$ we must take $m+(d-3) n=$ 0 . If $d>2$ this implies $m=0$, and therefore system (1.3) has no Darboux polynomials.

If $d=2$ then $n=m$. From equation (4.6) with $j=2 d-1=3$ we get the condition $C_{1}=-b m$. Now $F_{2}(u)=-b m u^{m+1}+\cdots$, where the dots mean here lower order terms. As $\operatorname{deg} F_{2} \leq m-2$, we must have $b=0$. We note that in the case $b=0$ the function $(u+a v)^{m}$, for each $m \in \mathbb{N}^{+}$, is a Darboux polynomial of system (5.3). Moreover further computations show that $C_{i}=0$ for all $i \geq 2$, which implies that there are no more 
Darboux polynomials than this straight line, as all the constants $C_{j}$ are fixed. Therefore statements (a) and (b) of the theorem follow.

We finally prove statement (c). The origin of system (1.3) is the only critical point and has index -1 when $a>0$ (it is a saddle point), therefore, the system has no periodic orbits for $a>0$.

When $a<0$, its index is +1 . Consider first the case $b=0$ and $d$ even. Then, the system has a reversible center at the origin by the Poincare reversibility criterion, because the point is monodromic and the system is invariant by the transformation $(x, y, t) \rightarrow$ $(-x, y,-t)$. Moreover, the periodic orbits surround the origin and form and unbounded set because there are no more critical points. It is easy to see that system (1.3) is a semicomplete family of rotated vector fields (SCFRVF) with respect to $b$, because

$$
y \frac{\partial}{\partial b}\left(a x+b y+x^{d-1} y\right)-\left(a x+b y+x^{d-1} y\right) \frac{\partial}{\partial b}(y)=y^{2} \geq 0,
$$

see $[12,30,31]$. One of the most useful properties of SCFRVF is the so-called nonintersection property. It says that if $\gamma_{\bar{b}}$ is a periodic orbit of the family when the parameter $b=\bar{b}$, it holds that $\gamma_{b_{1}} \cap \gamma_{b_{2}}=\emptyset$ when $b_{1} \neq b_{2}$. Hence, since for $b=0$ the periodic orbits of the center are unbounded, the system can not have periodic orbits for $b \neq 0$ (otherwise they would intersect the ones of the center).

Finally consider the case $d \geq 3$, odd. The divergence of the vector field associated to (1.3) is $b+x^{d-1}$. When $b \geq 0$ the above quantity is non-negative. Therefore, by the Bendixson criterion the system has no periodic orbits.

It remains to study the case $a<0, b<0$ and $d \geq 3$, odd. Precisely, this particular Liénard equation is under the hypotheses of the systems studied by Liénard, see [22]. He proved that the system has exactly one stable limit cycle. Afterwards, it was proved by Sansone that the limit cycle is hyperbolic, see [33]. All these results and much more information about Liénard systems can be found in [34]; see Theorem 2.4. Item (a) implies that the limit cycle is not algebraic.

\subsection{The Van der Pol differential system has no Darboux polynomials}

Because of its importance, we particularize here the above proof for the van der Pol system $(d=3)$. As in the previous section, we set $c=1$ in the expression of $V$. From equation (4.6) with $j=0$ we obtain $K_{0}(u)=k_{2}=n \in \mathbb{N} \cup\{0\}$ and $F_{0}(u)=u^{n}$. From equation (4.6) with $j=1$ we get $K_{1}(u)=k_{1}=0$ and $F_{1}(u)=C_{1} u^{n}$, with $C_{1}$ a constant. From equation (4.6) with $j=2$ we obtain $K_{2}(u)=k_{0}-m u=b n-m u$. We do not provide the expression of $F_{2}$ as it can be deduced from the general proof. At this moment we have $K(u, v)=n+(b n-m u) v^{d-1}$. 
From equation (4.6) with $j=3$ we get an expression for $F_{3}$ and no new conditions. We note that the cases $d+1, \ldots, 2 d-3$ of the proof of Theorem 1.1 do not appear here as $d=3$. Finally, from equation (4.6) with $j=4$ we have $R_{4}=\cdots+a m u^{n}+\cdots$. Since $a \neq 0$ we have $m=0$ and we have finished.

We have proved that the Van der Pol system has no Darboux polynomials in 5 steps, as we get $m=0$ from equation (4.6) with $j=2 d-2=4$. Hence we have provided a simple proof of the non-algebraicity of the van der Pol limit cycle different than the one appearing in [29].

Acknowledgments. The authors are partially supported by Spanish Government MTM2013-40998-P grant. The first author is additionally partially supported by grants Juan de la Cierva and MTM2009-14163-C0202. The second author is also supported by Generalitat de Catalunya Government 2014SGR568 grant.

\section{References}

[1] M.J. Ablowitz, A. Zeppetella, Explicit solutions of Fisher's equation for a special wave speed, Bull. Math. Biol. 41 (1979), 835-840.

[2] M.J. Álvarez, A. Ferragut, X. Jarque, A survey on the blow-up technique, Int. J. Bifur. Chaos Appl. Sci. Engrg. 21 (2011), 3103-3118.

[3] E. BEDFORD, K. KIM, Linear recurrences in the degree sequences of monomial mappings, Erg. Th. Dyn. Syst. 28 (2008), 1369-1375.

[4] R.I. Bogdanov, Bifurcation of the limit cycle of a family of plane vector fields, Selecta Math. Soviet 1 (1981), 373-387.

[5] J. Chavarriga, H. Giacomini, M. Grau, Necessary conditions for the existence of invariant algebraic curves for planar polynomial systems, Bull. Sci. Math. 129 (2005), 99-126.

[6] G. Chen, T. Ueta, Yet another chaotic attractor, Int. J. Bifur. Chaos 9 (1999), 1465-1466.

[7] G. CHÈzE, Darboux theory of integrability in the sparse case, J. Differ. Eq. 257 (2014), 601-609.

[8] C.J. Christopher, J. LlibRe, Integrability via invariant algebraic curves for planar polynomial differential systems, Ann. Differ. Eq. 16 (2000), 5-19.

[9] G. Darboux, Mémoire sur les équations différentielles algébriques du premier ordre et du premier degré (Mélanges), Bull. Sci. Math. 2 (1878), 60-96; 123-144; 151-200.

[10] G.T. DEE, W. VAN SAARloos, Bistable systems with propagating fronts leading to pattern formation, Phys. Rev. Lett. 60 (1988), 2641-2644. 
[11] M.V. Dolov, Limit cycles in the case of the center, Diff. Urav. 8 (1972), 16911692.

[12] G.F.D. DuFF, Limit-cycles and rotated vector fields, Ann. of Math. 67 (1953), 1531.

[13] R.A. FISHER, The wave of advance of advantageous genes, Ann. Eugenics 7 (1937), 355-369.

[14] I.A. GARcíA, Transcendental Limit Cycles Via the Structure of Arbitrary Degree Invariant Algebraic Curves of Polynomial Planar Vector Fields, Rocky Mountain J. Math. 35 (2005), 501-515.

[15] A. Gasull, H. Giacomini, Explicit traveling waves and invariant algebraic curves, preprint 2013.

[16] A. Gasull, H. Giacomini, J. Torregrosa, Explicit non-algebraic limit cycles for polynomial systems, J. Comput. Appl. Math. 200 (2007), 448-457.

[17] A. Gasull, H. Giacomini, J. Torregrosa, Explicit upper and lower bounds for the traveling wave solutions of Fisher-Kolmogorov type equations, Discrete Contin. Dyn. Syst. 33 (2013), 3567-3582.

[18] A. GoRIELY, Integrability and Nonintegrability of Dynamical Systems, Adv. Series in Nonlinear Dynamics 19, World Scientific 2001.

[19] B. Hasselblatt, J. Propp, Degree-growth of monomial maps, Erg. Th. Dynam. Sys. 27 (2007), 1375-1397.

[20] J.P. Jounnolou, Equations de Pfaff algébriques, Lectures Notes in Math. 708, Springer-Verlag, New York, 1979.

[21] A. Kolmogorov, I. Petrovskit, N. Piskunov, A study of the diffusion equation with increase in the amount of substance, and its application to a biological problem, In V. M. Tikhomirov, editor, Selected Works of A. N. Kolmogorov I, 248270. Kluwer 1991. Translated by V. M. Volosov from Bull. Moscow Univ., Math. Mech. 1 (1937), 1-25.

[22] A. LIÉNARD, Étude des oscillations entretenues, Revue génér. de l'électr. 23 (1928), 901-902; 906-954.

[23] J. Llibre, A. MAhDi, C. VAlls, Darboux integrability of the Lü system, J. Geom. Phys. 63 (2013), 118-128.

[24] J. Llibre, X. Zhang, Darboux integrability of real polynomial vector fields on regular algebraic hypersurfaces, Rend. circ. matem. Palermo 51 (2002), 109-126. 
[25] J. Llibre, X. Zhang, Invariant algebraic surfaces of the Lorenz system, J. Math. Phys. 43 (2002), 1622-1645.

[26] E.N. LorenZ, Deterministic nonperiodic flow, J. Atmos. Sci. 20 (1963), 130-141.

[27] J. Lü, G. CHEN, A new chaotic attractor coined, Internat. J. Bifur. Chaos 12 (2002), 659-661.

[28] T. LÜ, X. ZHANG, Darboux polynomials and algebraic integrability of the Chen system, Int. J. Bifur. Chaos 17 (2007), 2739-2748.

[29] K. OdAni, The limit cycle of the van der Pol Equation is Not Algebraic, J. Diff. Eq. 115 (1995), 146-152.

[30] L.M. PERKo, Global families of limit cycles of planar analytic systems, Trans. Amer. Math. Soc. 322, (1990) 627-656.

[31] L.M. PERKo, Differential equations and dynamical systems New York [etc.]: Springer-Verlag, (1996) 2nd ed.

[32] M. Pelletier, Éclatements quasi homogènes, Ann. Fac. Sci. Toulouse 4 (1995), 879-937.

[33] G. SAnsone, Sopra l'equazione di Liénard delle oscillazioni di rilassamento, Ann. Mat. Pura Appl. 28 (1949), 153-181.

[34] M. SabATini, G. Villari, On the uniquenesss of limit cycles for Liénards equation: the legacy of G. Sansone, Le Matematiche LXV (2010) Fasc. II, 201-214.

[35] A. SEIDENBERG, Reduction of singularities of the differential equation Ady = $B d x$, Amer. J. Math. 90, (1968) 248-269.

[36] F. TAKENS, Forced oscillations and bifurcations, Applications of global analysis I, Common Math. Inst. Rijksuniversitat Utrecht 3 (1974), 1-59.

[37] C. VALLs, Invariant algebraic surfaces for generalized Raychaudhuri equations, Commun. Math. Phys. 308 (2011), 133-146.

[38] J.A. WEIL, Constantes et polynômes de Darboux en algèbre différentielle: applications aux systèmes différentiels linéaires, Ph.D. Thesis, École Polytechnique, 1995.

[39] W. ZHOU, Y. XU, H. LU, L. PAN On dynamics analysis of a new chaotic attractor, Phys. Lett. A 372 (2008), 5773-5777. 